\title{
COMMENT
}

\section{Mining for therapeutic gold}

\author{
Francis S. Collins
}

\author{
A comprehensive and collaborative strategy to enable the investigation of new uses of \\ approved and abandoned drug compounds could advance translational research.
}

Discoveries about the molecular basis of disease are providing unprecedented opportunities to translate research into clinically useful products. However, the translational process is fraught with frustration: failure rates can be as high as $95 \%$, the average time from target selection to approval is $\sim 13$ years and, when failures are accounted for, the cost of bringing a new drug to market exceeds US $\$ 1$ billion $^{1}$. So, strategies to reduce the time frame, decrease costs and improve success rates are urgently needed.

Drug rescue and repurposing can be one of those strategies, and it offers the key advantage of harnessing previous research and development (R\&D) efforts. Approved drugs and many abandoned compounds have already been tested in humans, and so detailed information is available on their pharmacology, formulation, dosing and potential toxicity. This can enable the rapid testing of new clinical hypotheses, leading to remarkable health outcomes. For example, in the early days of the HIV epidemic, investigators at the US National Institutes of Health (NIH) collaborated with academic and industry experts to rescue AZT - a compound that was originally investigated for use in cancer but abandoned owing to lack of efficacy - and it became the first drug to treat patients with HIV.

Looking to the future, much could be gained by wider application of comprehensive collaborative approaches to drug rescue and repurposing. However, there are scientific, economic and administrative challenges that need to be addressed, including the collection and organization of compounds and data, incentives for further development and commercialization, safety issues and intellectual property considerations. The private sector holds a substantial proportion of the assets, data and knowledge needed for drug rescue and repurposing, but the ideas and wherewithal to advance new applications, especially for rare diseases, may come from different companies, the non-profit sector or academia.

To explore ways to approach drug rescue and repurposing more strategically and comprehensively, the NIH held a round-table meeting with leading representatives of academia, government and private sector R\&D on 21-22 April 2011. Acknowledging the value of drug rescue and repurposing, participants at the meeting discussed ways to make the process more practical and less burdensome (see page 399). Furthermore, it was agreed to establish a round table as a standing forum for fostering cross-sector efforts in translational science to tackle challenges for which such collaboration is critical for success.

Informed by these discussions and in close collaboration with industry, academia and non-profit organizations, the NIH will be launching a comprehensive effort to identify appropriate abandoned compounds, establish master agreements, match partners, make data and resources available, and provide a central access point to relevant resources and expertise. This will facilitate a full range of scientific approaches to drug rescue and repurposing - from serendipitous discovery of new applications, to targeted matching based on known mechanisms of action, to systematic screening using relevant assays drawn from multiple sectors. This latter option will be particularly useful for phenotypic screens in which the actual target is not known. There is also a key role for the US Food and Drug Administration (FDA) in advancing drug rescue and repurposing efforts (for example, for rare diseases ${ }^{2}$ ), and the recently established NIH-FDA Leadership Council could be a forum for exploring strategies and driving progress.

As one immediate step, the NIH will be augmenting activities that are underway to organize available data on drugs and investigational compounds through the $\mathrm{NIH}$ Chemical Genomics Center Pharmaceutical Collection (NPC) ${ }^{3}$. As a publicly accessible database and a full physical collection of small molecules that are approved for human use, the NPC is intended to aid collaboration by enabling high-throughput screening and drug repurposing efforts across a range of diseases. Such research will also be an important focus of the NIH's proposed National Center for Advancing Translational Sciences (NCATS). Indeed, drug rescue and repurposing research overall offers a key opportunity to learn from our collective past as we shape our future - a future in which translational science is more efficient and effective at delivering therapies and diagnostics to patients.

\footnotetext{
1. Paul, S. M. et al. Nature Rev. Drug Discov. 9, 203-214 (2010)

2. US FDA. http://www.fda.gov/Forlndustry/

DevelopingProductsforRareDiseasesConditions/

HowtoapplyforOrphanProductDesignation/ucm 216147.htm (2010).

3. Huang, R. et al. Sci. Transl. Med. 3, 80ps16 (2011).

Competing interests statement

The author declares no competing financial interests.
} 